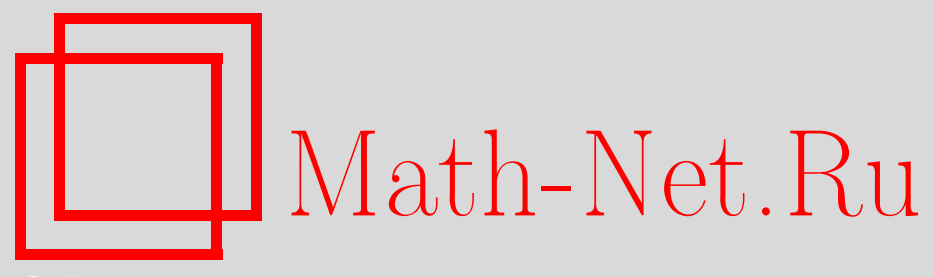

И. С. Борисов, А. А. Быстров, Построение стохастического интеграла от неслучайной функции без условия ортогональности интегрирующей меры, Теория вероятн. и ее примен., 2005, том 50, выпуск 1, 52-80

DOI: https://doi.org/10.4213/tvp158

Использование Общероссийского математического портала MathNet.Ru подразумевает, что вы прочитали и согласны с пользовательским соглашением

http://www . mathnet.ru/rus/agreement

Параметры загрузки:

IP: 34.239 .49 .27

26 апреля 2023 г., 13:10:08

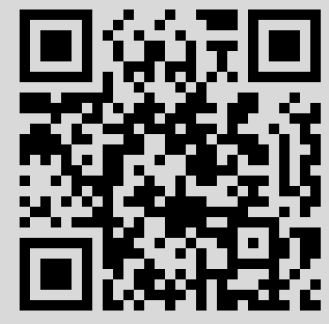




\section{ПОСТРОЕНИЕ СТОХАСТИЧЕСКОГО ИНТЕГРАЛА ОТ НЕСЛУЧАЙНОЙ ФУНКЦИИ БЕЗ УСЛОВИЯ ОРТОГОНАЛЬНОСТИ ИНТЕГРИРУЮЩЕЙ МЕРЫ ${ }^{1)}$}

В работе предложена конструкция абстрактного стохастического интеграла от неслучайной функции без классического требования ортогональности интегрируюшей стохастической меры. Конструкция включает в себя известные модели как одномерного, так и кратного стохастических интегралов. Условия существования этого интеграла конкретизированы для интегрирующих стохастических мер, порожденных случайными процессами с неортогональными приращениями из некоторых достаточно широких классов.

Ключевые слова и фразы: стохастический интеграл, кратный стохастический интеграл, элементарная стохастическая мера, гауссовские процессы, регулярное фрактальное броуновское движение.

1. Введение. При изучении случайных процессов, а также при описании некоторых распределений, возникающих в тех или иных приложениях стохастического анализа (например, в статистике), важную роль играют интегралы, записываемые в виде $\int f(t) d \xi(t)$ или $\int f\left(t_{1}, \ldots, t_{n}\right) d \xi\left(t_{1}\right) \cdots d \xi\left(t_{n}\right)$, где ядра $f(t)$ и $f\left(t_{1}, \ldots, t_{n}\right)$ - заданные неслучайные функции, а $\xi(t)$ - случайный процесс. Реализации процесса $\xi(t)$, вообще говоря, являются функциями неограниченной вариации, и интегралы указанного вида без каких-либо условий регулярности на ядра (скажем, лишь при условии их интегрируемости в смысле Лебега) нельзя понимать как интегралы Римана-Стилтьеса или ЛебегаСтилтьеса, существующие для почти всех реализаций $\xi(t)$.

Впервые стохастический интеграл от неслучайной функции по независимым прирашениям винеровского процесса рассмотрел в 1923 г. Н. Винер [1]. Основанное на технике гильбертовых пространств построение интегралов от неслучайных функций по стохастическим мерам,

* Институт математики им. С.Л. Соболева СО РАН, просп. Академика Коптюга, 4, 630090 Новосибирск, Россия; e-mail: sibam@math.nsc.ru, corpsegrinder@ngs.ru

1) Работа выполнена при финансовой поддержке проекта РФФИ 03-01-00459 и гранта INTAS 03-51-5018. 
порожденным случайными процессами с ортогональными (некоррелированными) приращениями, независимо предложено в 1940 г. А.Н. Колмогоровым [2] и Г. Крамером [3]. Кратные стохастические интегралы по ортогональным гауссовским стохастическим мерам рассматривали К. Ито [4] и П. Майор [5].

Один из первых результатов, касающихся интегрирования по неортогональным мерам, содержится в известной монографии М. Лоэва [6], где предложена конструкция стохастического интеграла Римана по приращениям произвольного гильбертова (т.е. с конечными вторыми моментами сечений) процесса на отрезке прямой. Распространение этой конструкции до лебеговой было осуществлено С. Камбанисом и С.Т. Хуангом в [7], где к тому же была рассмотрена схема построения кратного стохастического интеграла по приращениям произвольного гауссовского процесса. Условия, обеспечивающие корректное задание интегралов в этих работах, сводятся к проверке конечности некоторых детерминированных кратных интегралов по специальным мерам, построенным с помощью ковариационной функции интегрирующего случайного процесса. Причем даже в простейших случаях структура этой меры может оказаться весьма непростой, и проверка упомянутых условий может представлять собой отдельную проблему. В этом смысле весьма показательна работа А. Дасгупты и Г. Каллианпура [8], где как раз и «расшифровываются» условия Камбаниса-Хуанга в случае, когда интегрирующий процесс в конструкции кратного стохастического интеграла представляет собой регулярное фрактальное броуновское движение. Заметим, что в последнее время различным аспектам стохастического интегрирования по прирашениям этого гауссовского процесса посвящено немало работ (см., например, [9], [10]).

Отметим также, что в настояшей работе мы не касаемся вопросов построения стохастических интегралов в случае, когда ядро $f$ определенным образом зависит еще и от траектории интегрирующего случайного процесса (скажем, как в интегралах Ито, Стратоновича и др.). Это интенсивно развивающееся направление, тесно связанное с теорией стохастических дифференциальных уравнений, использует другой математический аппарат (как правило, для более узких классов интегрирующих процессов или ядер), который имеет мало общего с методологией настоящей работы.

Сначала напомним классическую конструкцию стохастического интеграла от неслучайной функции по случайному процессу с ортогональными прирашениями. При этом мы будем следовать наиболее общей схеме построения, изложенной в [11].

Пусть $\{\Omega, \mathscr{F}, \mathbf{P}\}-$ вероятностное пространство, $\mathscr{L}_{2}:=\mathscr{L}_{2}(\{\Omega$, $\mathscr{F}, \mathbf{P}\}), X$ - некоторое множество и $\mathfrak{M}$ - полукольцо подмножеств $X$ с единицей, т.е. $\mathfrak{M}$ замкнуто относительно операции «П», $X \in \mathfrak{M}$ и 
теоретико-множественная разность любых двух элементов из $\mathfrak{M}$ представима в виде конечного объединения элементов из $\mathfrak{M}$. Предположим, что на $\{\Omega, \mathscr{F}, \mathbf{P}\}$ задан случайный процесс $\{\mu(A) ; A \in \mathfrak{M}\}$ с конечными вторыми моментами сечений (т.е. $\mu(A) \in \mathscr{L}_{2}$; такие процессы иногда называют гильбертовыли), удовлетворяющий следующим дополнительным условиям:

(M1) $\mu\left(A_{1} \cup A_{2}\right)=\mu\left(A_{1}\right)+\mu\left(A_{2}\right)$ почти наверное, если $A_{1} \cap A_{2}=\varnothing$ и $A_{1} \cup A_{2} \in \mathfrak{M}$;

(M2) $\mathbf{E} \mu\left(A_{1}\right) \mu\left(A_{2}\right)=m\left(A_{1} \cap A_{2}\right)$, где $m(A)$ - некоторая $\sigma$ аддитивная конечная мера на $\sigma(\mathfrak{M})-$ минимальной $\sigma$-алгебре, порожденной элементами из $\mathfrak{M}$.

О п р е д е л е н и е 1 . Гильбертов случайный процесс $\{\mu(A)$; $A \in \mathfrak{M}\}$, удовлетворяющий условию (M1), называется элементарной стохастической мерой (при выполнении еще и (М2) - ортогональной), a $m(A)$ называется ее структурной функцией.

Отметим, что множество элементарных исходов полной меры, для которого имеет место условие (M1), вообще говоря, зависит от $A_{1}$ и $A_{2}$. При этом может и не существовать универсального множества полной меры в $\Omega$, для каждого элемента которого условие (M1) выполняется при всех $A_{1}, A_{2} \in \mathfrak{M}$, удовлетворяюших приведенным выше требованиям. Так что при выполнении (M1) реализации случайной функции множества $\{\mu(A) ; A \in \mathfrak{M}\}$ не обязаны быть аддитивными зарядами на $\mathfrak{M}$.

Свойство ортогональности стохастической меры (М2) означает, что если $A_{1} \cap A_{2}=\varnothing$, то случайные величины $\mu\left(A_{1}\right)$ и $\mu\left(A_{2}\right)$ ортогональны в $\mathscr{L}_{2}$. Типичными примерами ортогональных стохастических мер являются центрированные пуассоновские точечные процессы, а также абстрактные винеровские процессы $\left\{W_{\lambda}(A) ; A \in \mathfrak{M}\right\}$, т.е. центрированные стохастические гауссовские меры с произвольной структурной функцией $m(\cdot)=\lambda(\cdot)$. В последнем примере для любого конечного набора попарно несовместных множеств $A_{i} \in \mathfrak{M}, i=1, \ldots, n$, гауссовский вектор $\left(W_{\lambda}\left(A_{1}\right), \ldots, W_{\lambda}\left(A_{n}\right)\right)$ состоит из независимых компонент. Точно таким же свойством обладают соответствующие конечномерные проекции и пуассоновских точечных процессов. Отметим, что для произвольных полуколец $\mathfrak{M}$ существование абстрактных винеровских процессов на соответствующем вероятностном пространстве следует из классической теоремы А. Н. Колмогорова о согласованных распределениях. Основное свойство (М1) для этого процесса следует из элементарного тождества

$$
\mathbf{E}\left(W_{\lambda}(A \cup B)-W_{\lambda}(A)-W_{\lambda}(B)\right)^{2}=0,
$$

которое легко проверяется для любых несовместных $A$ и $B$. 
Далее, обозначим через $\mathscr{L}_{0}\{\mathfrak{M}\}$ следующий класс простых функций $f(x)$ :

$$
f(x)=\sum_{k=1}^{n} c_{k} I_{A_{k}}(x), \quad A_{k} \in \mathfrak{M}, k=1, \ldots, n, \quad A_{i} \cap A_{j}=\varnothing, i \neq j,
$$

где $n$ - любое натуральное число, $c_{k}$ - произвольные числа, а $I_{A}(x)$ индикатор множества $A$.

Определим стохастический интеграл от функции $f(x) \in \mathscr{L}_{0}\{\mathfrak{M}\}$ по элементарной стохастической мере $\mu(\cdot)$ формулой

$$
\eta(f):=\int f(x) \mu(d x)=\sum_{k=1}^{n} c_{k} \mu\left(A_{k}\right) .
$$

Теперь рассмотрим линейную оболочку $\mathscr{L}_{0}\{\mu\}$ уже введенного семейства случайных величин $\{\mu(A) ; A \in \mathfrak{M}\}$, т.е. множество случайных величин, представимых в виде $(2)$, и пространство $\mathscr{L}_{2}\{\mu\}$, являющееся замыканием. $\mathscr{L}_{0}\{\mu\}$ в гильбертовом пространстве случайных величин $\mathscr{L}_{2}$. Кроме того, обозначим символом $\mathscr{L}_{2}\{\mathfrak{M}\}$ замыкание $\mathscr{L}_{0}\{\mathfrak{M}\}$ в гильбертовом пространстве $\mathscr{L}_{2}(X, \sigma(\mathfrak{M}), m)$. В классической конструкции ортогональность используется следующим образом: соотношение (2) устанавливает изометрический изоморфизм (изометрию) $\eta(f)$ между $\mathscr{L}_{0}\{\mathfrak{M}\}$ и $\mathscr{L}_{0}\{\mu\}$, поскольку

$$
\|\eta(f)\|_{\mathscr{L}_{2}}^{2} \equiv \mathbf{E} \eta^{2}(f)=\sum_{k=1}^{n} c_{k}^{2} m\left(A_{k}\right)=\int f^{2}(x) m(d x)
$$

Это соответствие может быть продолжено до изометрии $\eta$ между $\mathscr{L}_{2}\{\mathfrak{M}\}$ и $\mathscr{L}_{2}\{\mu\}$ в силу полноты обоих пространств. Тогда для любой функции $f \in \mathscr{L}_{2}\{\mathfrak{M}\}$ полагаем по определению $\int f(x) \mu(d x)=\eta(f)$.

Заметим, что требование ортогональности не является необходимым для построения пространства функций, изометричного $\mathscr{L}_{2}\{\mu\}$. Так, в [9] приводится следующее утверждение.

Теорема А. Пусть Я -множество функиий, заданньх на вещественной прямой и удовлетворяющих следующим условиям:

1) $\mathscr{F}$ - евклидово пространство со скалярныл произведениèм $(f, g) \mathscr{F}$

2) $\mathscr{L}_{0}\{\mathfrak{M}\} \subset \mathscr{F} u(f, g)_{\mathscr{F}}=\mathbf{E} \eta(f) \eta(g)$ для всех $f, g \in \mathscr{L}_{0}\{\mathfrak{M}\}$;

3) множество $\mathscr{L}_{0}\{\mathfrak{M}\}$ плотно в $\mathscr{F}$ в норме, порожденной указанньим скалярньм произведением.

Тогда существует изометрия между пространством Я尹F и некоторьмм линейньм подпространством в $\mathscr{L}_{2}\{\mu\}$, являющаяся расширением отображения $f \mapsto \eta(f), f \in \mathscr{L}_{0}\{\mathfrak{M}\}$. Более того, $\mathscr{F}$ изометрично всему пространству $\mathscr{L}_{2}\{\mu\}$ тогда и только тогда, когда $\mathscr{F}$ полно. 
Наша цель - определить стохастический интеграл в максимальной общности так, чтобы эта схема включала в себя известные конструкции как одномерных, так и кратных стохастических интегралов с интегрирующими элементарными стохастическими (не обязательно гауссовскими!) мерами без условия ортогональности (М2). Кроме того, мы выделим классы интегрирующих случайных процессов, для которых сравнительно легко проверяются условия, обеспечивающие существование этого стохастического интеграла. Важным в предлагаемом подходе является то обстоятельство, что для корректного определения стохастического интеграла вместо упомянутой изометрии между введенными выше замкнутыми (в той или иной топологии) линейными оболочками достаточно построить вложение соответствующих функциональных пространств.

2. Основные определения и результаты. Пусть $\mu$ - элементарная стохастическая мера, удовлетворяющая только условию (M1). Определим на канонических прямоугольниках $A \times B$ декартова квадрата $X^{2}$, где $A, B \in \mathfrak{M}$, следующую функцию множества:

$$
m(A \times B):=\mathbf{E} \mu(A) \mu(B) .
$$

Мы рассматриваем $m$ как функцию множества, заданную на полукольце подмножеств $\mathfrak{M}^{2}:=\{A \times B ; A, B \in \mathfrak{M}\}$ с элементом $X^{2}$ в качестве единицы. Эта функция (вообще говоря, знакопеременная) аддитивна в следующем смысле: если $A=\bigcup_{i \leqslant n} A_{i}$ и $B=\bigcup_{j \leqslant r} B_{j}$, причем $A, B, A_{i}$, $B_{j} \in \mathfrak{M}$ и каждый из конечных наборов $\left\{A_{i}\right\}$ и $\left\{B_{j}\right\}$ состоит из попарно непересекающихся подмножеств, то $m(A \times B)=\bigcup_{i, j} m\left(A_{i} \times B_{j}\right)$. Кроме того, функция $m$ обладает очевидной симметрией: $m(A \times B)=m(B \times A)$.

Основное предположение. Функиия множества $\mathrm{m}(\cdot)$ есть конечная $\sigma$-аддитивная знакопеременная мера (заряд) на $\mathfrak{M}^{2}$, т.е. указанное выше свойство аддитивности имеет место и для любых счетных семейств подмножеств $\left\{A_{i}\right\} u\left\{B_{j}\right\}$.

В силу классической теоремы о продолжении меры (см., например, [12]) конечный заряд $m$ при выполнении основного предположения может быть продолжен на $\sigma\left(\mathfrak{M}^{2}\right)$ - минимальную $\sigma$-алгебру, содержащую все канонические прямоугольники пространства $X^{2}$. Этот заряд будем называть ковариационной мерой.

Пусть $Q^{+}$и $Q^{-}$- соответственно множества положительности и отрицательности ковариационной меры $m$. Поскольку функция $m$ конечна, то она (как и любой конечный заряд) допускает разложение Жордана: $m=m^{+}-m^{-}$, где $m^{+}$и $m^{-}$- неотрицательные $\sigma$-аддитивные функции множества (т.е. обычные меры на $\sigma\left(\mathfrak{M}^{2}\right)$ ), называемые соответственно положительной и отрицательной частями $m$; при этом $m^{+}(A)=m\left(A \cap Q^{+}\right) \geqslant 0$ и $m^{-}(A)=-m\left(A \cap Q^{-}\right) \geqslant 0$ для любого $A \in \mathfrak{M}^{2}$. 
Нетрудно понять, что вместе с зарядом $m$ его положительная и отрицательная части будут также симметричньми мерами, поскольку каждое из множеств $Q^{+}$и $Q^{-}$будет симметричным относительно главной диагонали. Стало быть, функция $|m|=m^{+}+m^{-}$, называемая полной вариацией заряда $m$, обладает всеми свойствами обычной конечной симметричной меры.

Введем в рассмотрение также проекцию меры $|m|$ на одну из осей: $\bar{m}(A):=|m|(A, X)=|m|(X, A)$. Понятно, что $\bar{m}$ - конечная $\sigma$-аддитивная мера на $\sigma(\mathfrak{M})$.

О п р е д е л е н и е 2. Будем говорить, что мера $|m|$ неотрицательно определена, если

$$
\int_{X^{2}} f(t) f(s)|m|(d t, d s) \geqslant 0
$$

для любой $\bar{m}$-интегрируемой $\sigma(\mathfrak{M})$-измеримой функции $f$.

Мера $|m|$ положительно определена, если при тех же условиях знак неравенства в $(3)$ будет строгим для любой ненулевой $(\bmod \bar{m})$ функции $f$.

Легко видеть, что сама ковариационная мера $m$ всегда неотрицательно определена, поскольку для любой ступенчатой функции $f \in \mathscr{L}_{0}(\mathfrak{M})$ имеем

$$
\int_{X^{2}} f(t) f(s) m(d t, d s)=\mathbf{E}\left(\int f(t) \mu(d t)\right)^{2} .
$$

Так что левая часть этого тождества останется неотрицательной для любой $\sigma(\mathfrak{M})$-измеримой $\bar{m}$-интегрируемой функции $f$ в силу определения интеграла Лебега (для компонент $m^{+}$и $m^{-}$) и теоремы Фубини.

Однако отсюда вовсе не следует, что меры $m^{+}$и $m^{-}$, а значит, и $|m|$ наследуют указанное свойство. Соответствующий контрпример содержится в предложении 2.

Отметим также, что если меры $\mathrm{m}^{+}$и $\mathrm{m}^{-}$неотрицательно определены, а хотя бы одна из них положительно определена, то и мера $|\mathrm{m}|$ будет положительно определенной. Например, таковой будет полная вариация ковариационной меры абстрактного броуновского моста, т.е. элементарной центрированной гауссовской меры

$$
W_{\lambda}^{0}(A):=W_{\lambda}(A)-\lambda(A) W_{\lambda}(X), \quad A \in \mathfrak{M},
$$

имеющей ковариационную меру $m(A \times B)=\lambda(A \cap B)-\lambda(A) \lambda(B)$. В этом случае мера $m^{+}(A \times B)=\lambda(A \cap B)$ положительно определена, a $m^{-}(A \times B)=\lambda(A) \lambda(B)$ - неотрицательно. Таким же свойством обладает и регулярное фрактальное броуновское движение, для которого 
$m^{-} \equiv 0$, a $m^{+}$положительно определена (см. далее соответствующий пример в п. 3.4).

Всюду в дальнейшем, когда $m$ выступает в качестве интегрирующей меры под знаком интеграла, мы будем следовать более традиционной записи $m(d t, d s)$ вместо $m(d t \times d s)$ и соответственно $|m|(d t, d s)$ вместо $|m|(d t \times d s)$.

Как и прежде, обозначим через $\mathscr{L}_{0}\{\mathfrak{M}\}$ класс всех простых функций вида (1). Определим стохастический интеграл от простой функции по формуле (2). Множество случайных величин, являющихся интегралами от функций из $\mathscr{L}_{0}\{\mathfrak{M}\}$, также обозначим $\mathscr{L}_{0}\{\mu\}$. Квадрат расстояния в пространстве $\mathscr{L}_{2}$ между случайными величинами $\eta\left(f_{l}\right)$ и $\eta\left(f_{n}\right)$ для любых элементов $f_{l}, f_{n} \in \mathscr{L}_{0}\{\mathfrak{M}\}$ можно записать так:

$$
\begin{aligned}
\mathbf{E}\left(\eta_{l}-\eta_{n}\right)^{2} & =\mathbf{E}\left(\int f_{l}(t) \mu(d t)-\int f_{n}(t) \mu(d t)\right)^{2} \\
& =\mathbf{E}\left(\sum_{k=1}^{l} c_{k} \mu\left(A_{k}\right)-\sum_{k=1}^{n} d_{k} \mu\left(B_{k}\right)\right)^{2},
\end{aligned}
$$

где $A_{k}, B_{k} \in \mathfrak{M}$, а $c_{k}, d_{k} \in \mathbf{R}$. Так как $\mathfrak{M}-$ полукольцо, то любую пару функций вида (1) можно представить как линейные комбинации индикаторов одних и тех же множеств из $\mathfrak{M}$, порождающих с помощью операции $\cup$ два вышеупомянутых разбиения. Обозначим указанную систему порождающих через $\left\{C_{k}\right\}$. Тогда имеем

$$
\begin{aligned}
\mathbf{E}\left(\eta_{l}-\eta_{n}\right)^{2} & =\mathbf{E}\left(\sum_{k} c_{k}^{n, l} \mu\left(C_{k}\right)\right)^{2}=\sum_{k} \sum_{j} c_{k}^{n, l} c_{j}^{n, l} \mathbf{E} \mu\left(C_{k}\right) \mu\left(C_{j}\right) \\
& =\sum_{k} \sum_{j} c_{k}^{n, l} c_{j}^{n, l} m\left(C_{k} \times C_{j}\right) \\
& =\int_{X^{2}}\left(f_{n}(t)-f_{l}(t)\right)\left(f_{n}(s)-f_{l}(s)\right) m(d t, d s) .
\end{aligned}
$$

В связи с представлением (5) введем в рассмотрение следующее пространство $\sigma(\mathfrak{M})$-измеримых функций:

$$
S:=\left\{f: \int_{X^{2}} f(t) f(s) m(d t, d s)<\infty\right\} .
$$

Отметим, что существование кратного интеграла в этом определении эквивалентно существованию соответствующих интегралов по мерам $m^{+}$и $m^{-}$.

Кроме того, введем в рассмотрение заданный на $S^{2}$ билинейный функционал

$$
(f, g):=\int_{X^{2}} f(t) g(s) m(d t, d s),
$$


для которого выполнены все аксиомы скалярного произведения за исключением одной: уравнение $(f, f)=0$ имеет, вообще говоря, не единственное решение. Последнее будет иметь место, если для некоторого набора попарно непересекающихся подмножеств $\left\{A_{k}\right\}$ случайные величины $\left\{\mu\left(A_{k}\right)\right\}$ в (4) являются линейно зависимыми с вероятностью 1. Скажем, это будет так для обобщенного броуновского моста $W_{\lambda}^{0}(A)$. Так что функционал $\|f\|:=\sqrt{(f, f)}$ образует в $S$ полунорму. Однако если провести факторизацию $S$ по множеству корней уравнения $\|f\|=0$ (т.е. условиться не различать элементы из $S$, разность которых будет одним из указанных корней), то можно говорить об $\|\cdot\|$ как о норме. Именно это в дальнейшем мы и будем иметь в виду, называя $\|\cdot\|$ нормой.

Отметим, что соотношения (2) и (5) задают изометрию между $\left\{\mathscr{L}_{0}\{\mathfrak{M}\},\|\cdot\|\right\}$ и $\mathscr{L}_{0}\{\mu\}$. Нетрудно также установить, что замыкание в норме $\|\cdot\|$ в $S$ линейной оболочки $\mathscr{L}_{0}\{\mathfrak{M}\}$ совпадает с $S$, но при этом $S$ может не быть полным.

Как и прежде, обозначим через $\mathscr{L}_{2}\{\mu\}$ замыкание $\mathscr{L}_{0}\{\mu\}$ в гильбертовом пространстве случайных величин $\mathscr{L}_{2}$. Заметим, что если $S$ не полно, то пространства $(S,\|\cdot\|)$ и $\mathscr{L}_{2}\{\mu\}$ не являются изометричными (см. теорему A). Однако всегда имеет место вложение пространств: для всякой функции $f \in S$ найдется $\eta(f) \in \mathscr{L}_{2}(\mu)$ (см. далее теорему 1 ). Тогда можно положить стохастический интеграл от функции $f$ из $S$ равным $\eta(f)$.

В частном случае регулярного фрактального броуновского движения пространство $S$ фигурировало в [9], где оно обозначалось $\left(|\Lambda|^{k},\|\cdot\|_{|\Lambda|^{k}}\right)$. Там же было замечено, что это пространство является неполным. Кроме того, несколько более узкие, чем $S$, подпространства ядер при построении стохастических интегралов были введены в [6] и [7].

Теперь мы попытаемся построить в $S$ другую норму, в известном смысле более конструктивную, чем $\|\cdot\|$, которая также позволит осуществить интересующее нас вложение пространств, а для некоторых классов ковариационных мер еще и обеспечить полноту пространства $S$. Последнее обстоятельство весьма важно в вопросах обратимости линейного оператора $\eta(\cdot): S \rightarrow \mathscr{L}_{2}(\mu)$.

Рассмотрим функционал

$$
d(f, g):=\int_{X^{2}}|f(t)||g(s)||m|(d t, d s)
$$

Заметим, что в силу конечности меры $m$ и определения интеграла Лебега неравенства $d(f, f)<\infty$ и $\|f\|<\infty$ эквивалентны. Стало быть, пространство $S$ можно определить условием $d(f, f)<\infty$.

Предложение 1. Функционал

$$
d_{0}(f):=\sqrt{d(f, f)}
$$


является нормой в $S$ тогда и только тогда, когда выполнено условие (3).

Д о к а з а т е л ь с в о. Пусть выполнено условие (3). Тогда билинейный функционал

$$
(f, g)_{*}:=\int_{X^{2}} f(t) g(s)|m|(d t, d s)
$$

обладает основными свойствами скалярного произведения в $S$ (он будет скалярным произведением лишь в случае, когда мера $|m|$ положительно определена). В частности, функционал $d_{0}(f)$ представляет собой полунорму. Стало быть,

$$
d_{0}(f+g) \leqslant \sqrt{(|f|+|g|,|f|+|g|)_{*}} \leqslant d_{0}(f)+d_{0}(g)
$$

т.е. для $d_{0}(\cdot)$ выполнено неравенство треугольника. Выполнение остальных аксиом нормы очевидно.

Пусть теперь условие (3) не выполнено, т.е. найдется ненулевая относительно меры $\bar{m}$ функция $f_{0}$ такая, что $\left(f_{0}, f_{0}\right)_{*}<0$. Положим $f_{0}^{+}:=\max \{0, f(x)\}, f_{0}^{-}:=\max \{0,-f(x)\}$ и рассмотрим квадратный трехчлен

$$
R(x):=\left(f_{0}^{+}-x f_{0}^{-}, f_{0}^{+}-x f_{0}^{-}\right)_{*} .
$$

Тогда

$$
R(1)=\left(f_{0}^{+}-f_{0}^{-}, f_{0}^{+}-f_{0}^{-}\right)_{*}=\left(f_{0}, f_{0}\right)_{*}<0 \text {. }
$$

Поскольку коэффициент $\left(f_{0}^{-}, f_{0}^{-}\right)_{*}$ при $x^{2}$ квадратного трехчлена $R(x)$ положителен (в силу условия $\left(f_{0}, f_{0}\right)_{*}<0$ обе компоненты $f_{0}^{+}$и $f_{0}^{-}$положительны на множествах положительной меры $\bar{m}$ ), то отсюда с необходимостью следует положительность дискриминанта $R(x)$, т.е. неравенство

$$
\left(f_{0}^{+}, f_{0}^{-}\right)_{*}^{2}>\left(f_{0}^{+}, f_{0}^{+}\right)_{*}\left(f_{0}^{-}, f_{0}^{-}\right)_{*} .
$$

Заметим, что это условие («контрнеравенство» Коши-Буняковского) влечет за собой нарушение неравенства треугольника для $d_{0}(\cdot)$ на указанных элементах. Действительно,

$$
\begin{array}{r}
d_{0}^{2}\left(f_{0}^{+}+f_{0}^{-}\right)=\left(f_{0}^{+}+f_{0}^{-}, f_{0}^{+}+f_{0}^{-}\right)_{*}=\left(f_{0}^{+}, f_{0}^{+}\right)_{*}+\left(f_{0}^{-}, f_{0}^{-}\right)_{*}+2\left(f_{0}^{+}, f_{0}^{-}\right)_{*} \\
>\left(f_{0}^{+}, f_{0}^{+}\right)_{*}+\left(f_{0}^{-}, f_{0}^{-}\right)_{*}+2 \sqrt{\left(f_{0}^{+}, f_{0}^{+}\right)_{*}\left(f_{0}^{-}, f_{0}^{-}\right)_{*}}=\left(d_{0}(f)+d_{0}(g)\right)^{2},
\end{array}
$$

т.е. функционал $d_{0}(\cdot)$ не является нормой. Предложение доказано.

Примеры проверки условия (3) будут приведены ниже. Покажем теперь, что это условие не всегда выполнено. 
Предложение 2. Пусть $\xi_{1}, \xi_{2}$ - независимье случайные величины с нулевьми средними и единичньли дисперсиями. Рассмотрим на отрезке $[0,1]$ случайньй прочесс

$$
\xi(t)=\xi_{1} \cos t+\xi_{2} \sin t .
$$

Тогда мера $|m|$, построенная по ковариачионной функиии этого проиесса, не удовлетворяет условию (3).

Д о к а з а т е л ь с т в о. Имеем по определению для любых $t, s \in \mathbf{R}$ и $\Delta_{1}, \Delta_{2} \downarrow 0$

$$
\begin{aligned}
& m\left(\left(t, t+\Delta_{1}\right] \times\left(s, s+\Delta_{2}\right]\right)=\mathbf{E}\left(\xi\left(t+\Delta_{1}\right)-\xi(t)\right)\left(\xi\left(s+\Delta_{2}\right)-\xi(s)\right) \\
& =\left(\sin \left(t+\Delta_{1}\right)-\sin t\right)\left(\sin \left(s+\Delta_{2}\right)-\sin s\right) \\
& \quad+\left(\cos \left(t+\Delta_{1}\right)-\cos t\right)\left(\cos \left(s+\Delta_{2}\right)-\cos s\right) \\
& =(\cos t \cos s+\sin t \sin s) \Delta_{1} \Delta_{2}+o\left(\Delta_{1} \Delta_{2}\right) \\
& =\cos (t-s) \Delta_{1} \Delta_{2}+o\left(\Delta_{1} \Delta_{2}\right) .
\end{aligned}
$$

Таким образом, мера $m$, а также и мера $|m|$ абсолютно непрерывны относительно двумерной меры Лебега $\lambda$, причем соответствующая производная Радона-Никодима вычисляется по формуле

$$
\frac{d|m|}{d \lambda}(t, s)=|\cos (t-s)| \text {. }
$$

Предположим, что мера $|m|$ неотрицательно определена в смысле определения (3). Это означает, что функция $f(x)=|\cos x|$ положительно определена в классическом смысле:

$$
\sum_{i, j=1}^{n} f\left(t_{i}-t_{j}\right) z_{i} z_{j} \geqslant 0
$$

для всех натуральных $n$, а также всех $t_{i}$ и $z_{i} \in \mathbf{R}$. Заметим, что $f$ непрерывна и $f(0)=1$. Таким образом, согласно теореме Бохнера функция $f(x)=|\cos x|$ с необходимостью является характеристической функцией, чего, как нетрудно видеть, не может быть. Стало быть, предположение о том, что мера $|m|$ удовлетворяет условию (3), неверно. Предложение доказано.

Таким образом, при выполнении условия (3) наряду с нормой $\|\cdot\|$ в пространстве $S$ можно рассматривать более сильную норму $d_{0}(\cdot)$ (неравенство $\|f\| \leqslant d_{0}(f)$ очевидно). Отметим, что даже в случае знакопостоянной ковариационной меры (в этом случае условие (3) выполнено, т.е. функционал $d_{0}$ - норма) сходимость в $\|\cdot\|$-норме, вообще говоря, не влечет $d_{0}$-сходимость. Например, сказанное имеет место для норм, построенных по ковариационной мере регулярного фрактального броуновского движения (см. [9]). 
Предложение 3. Пусть выполнено условие (3) и существует такая мера $m_{0}$ на $\sigma(\mathfrak{M})$, что $|m|(A \times B) \geqslant m_{0}(A) m_{0}(B)$ для любых $A, B \in \sigma(\mathfrak{M})$. Тогда пространство $\left(S, d_{0}\right)$ банахово.

Д о к а за т е ль с т в о. В силу условий на меру $|m|$ для любой функции $h$ из $S$ имеем

$$
d_{0}^{2}(h)=\int_{X^{2}}|h(t)||h(s)||m|(d t, d s) \geqslant\left(\int_{X}|h(t)| m_{0}(d t)\right)^{2}=\|h\|_{1}^{2},
$$

где $\|\cdot\|_{1}-$ норма банахова пространства $\mathscr{L}_{1}\left(X, m_{0}\right)$, т.е. линейное нормированное пространство $\left(S, d_{0}\right)$ вкладывается в банахово пространство $\mathscr{L}_{1}\left(X, m_{0}\right)$. Пусть $\left\{f_{n}\right\}-$ фундаментальная последовательность функций в $\left(S, d_{0}\right)$, а значит, и в $\mathscr{L}_{1}\left(X, m_{0}\right)$. В силу полноты пространства $\mathscr{L}_{1}\left(X, m_{0}\right)$ сушествует предел $f:\left\|f_{n}-f\right\|_{1} \rightarrow 0$ при $n \rightarrow \infty$. Но тогда (см. [12]) найдется подпоследовательность $f_{n_{l}}$, сходящаяся к $f$ почти всюду относительно $m_{0}$. Наконец, по теореме Фату имеем

$$
\begin{aligned}
d_{0}(f) & =d_{0}\left(\liminf _{l} f_{n_{l}}\right) \leqslant \liminf _{l} d_{0}\left(f_{n_{l}}\right)<\infty, \\
d_{0}\left(f-f_{n}\right) & \leqslant d_{0}\left(\liminf _{l} f_{n_{l}}-f_{n}\right) \leqslant \liminf _{l} d_{0}\left(f_{n_{l}}-f_{n}\right) \longrightarrow 0
\end{aligned}
$$

при $n, m \rightarrow \infty$. Таким образом, функция $f$ является пределом фундаментальной в $\left(S, d_{0}\right)$ последовательности $\left\{f_{n}\right\}$. Предложение доказано.

При нарушении условия (3) можно рассмотреть еще одно вложение. Введем в рассмотрение гильбертово пространство $\overline{\mathscr{L}}_{2}:=$ $\mathscr{L}_{2}(X, \sigma(\mathfrak{M}), \bar{m})$. Заметим, что построенное гильбертово пространство не является изометричным пространству $\mathscr{L}_{2}\{\mu\}$ : условие 2) теоремы А не выполняется, так как на множестве функций $f \in \overline{\mathscr{L}}_{2}$ имеет мес'го лишь неравенство

$$
\|f\|^{2} \leqslant(f, f)_{\overline{\mathscr{L}}_{2}}
$$

которое является прямым следствием неравенства Коши-Буняковского и теоремы Фубини. Стало быть, пространство $\overline{\mathscr{L}}_{2}$ вкладывается в $(S,\|\cdot\|)$ (или в $\left(S, d_{0}\right)$ при выполнении условия $\left.(3)\right)$. Несмотря на то, что пространство $\overline{\mathscr{L}}_{2}$ существенно у́же $(S,\|\cdot\|)$ (или $\left.\left(S, d_{0}\right)\right)$, принадлежность той или иной функции к $\overline{\mathscr{L}}_{2}$, как правило, проверяется значительно проще, чем принадлежность к двум вышеупомянутым пространствам.

Справедлива следующая теорема.

Теорема 1. Пусть выполнено одно из следуюших трех условий:

1) $\left\{f_{n}\right\}$ - последовательность бункиий вида (1), сходящаяся $\kappa f \in S$ в норме $\|\cdot\|$;

2) выполнено условие (3) $и\left\{f_{n}\right\}-$ последовательность функиий из $S$ вида (1), сходячаяся $\propto f \in S$ в норме $d_{0}$;

3) $\left\{f_{n}\right\}-$ последовательность функиий вида (1), сходящаяся $\kappa f \in \overline{\mathscr{L}}_{2}$ в норме $\overline{\mathscr{L}}_{2}$. 
Тогда для последовательности случайньх величин $\left\{\eta\left(f_{n}\right)\right\}$ вида (2) существует $\mathscr{L}_{2}$-предел $\eta(f)$, который не зависит от выбора последовательности $\left\{f_{n}\right\}$.

Д о к а зат е л ь с т о. Сначала докажем существование последовательности ступенчатых функций вида (1), сходящихся к $f$ в одной из двух введенных норм пространства $S$ (т.е. в условиях пунктов 1 ) и 2) теоремы). Аналогичный результат для гильбертова пространства $\overline{\mathscr{L}}_{2}$ (пункт 3$)$ ) хорошо известен (см. [11]). Обозначим через $\alpha(\mathfrak{M})$ минимальную алгебру, содержащую все элементы из $\mathfrak{M}$, т.е. к полукольцу $\mathfrak{M}$ мы добавляем всевозможные конечные объединения подмножеств из класса $\mathfrak{M}$ и их дополнений. Стало быть, классы всех ступенчатых функций, построенных по разбиениям с элементами из $\mathfrak{M}$ или из $\alpha(\mathfrak{M})$, совпадают. Поэтому мы можем искать требуемую последовательность ступенчатых функций среди функций вида

$$
f_{n}(t)=\sum_{k=1}^{n} a_{k}^{n} I_{B_{k}^{n}}(t), \quad B_{k}^{n} \in \alpha(\mathfrak{M}) .
$$

Пусть сначала $0 \leqslant f(t)<M$. Обозначим

$$
A_{k}^{n}:=\left\{t \in X: \frac{M(k-1)}{n} \leqslant f(t)<\frac{M k}{n}\right\} .
$$

Понятно, что $A_{k}^{n} \in \sigma(\mathfrak{M})$ (причем эти множества могут не принадлежать алгебре $\alpha(\mathfrak{M}))$. Тогда последовательность функций $g_{n}(t):=$ $\sum_{k=1}^{n}(M k / n) I_{A_{k}^{n}}(t)$ приближает $f$ в равномерной метрике, а значит, и в упомянутых двух метриках пространства $S$ (в силу конечности меры $|m|)$. Введем в $\sigma(\mathfrak{M})$ полуметрику по формуле $\rho(A, B)=$ $\bar{m}(A \bar{B} \cup \bar{A} B)$. Тогда в силу известных результатов (см., например, [13]) $\sigma(\mathfrak{M})$ есть замыкание $\alpha(\mathfrak{M})$ в этой полуметрике. Значит, для любых $\delta>0$ и $A \in \sigma(\mathfrak{M})$ найдется такое $B \in \alpha(\mathfrak{M})$, что $\rho(A, B)<\delta$.

Далее, для каждого $A_{k}^{n}$ выберем $B_{k}^{n} \in \alpha(\mathfrak{M})$ так, чтобы $\rho\left(B_{k}^{n}, A_{k}^{n}\right)<n^{-2}$. Рассмотрим теперь последовательность ступенчатых функций $f_{n}(t):=\sum_{k=1}^{n}(M k / n) I_{B_{k}^{n}}(t)$; при этом конечный набор подмножеств $\left\{B_{k}^{n}\right\}$ не обязательно представляет собой разбиение выборочного пространства. Докажем, что $f_{n}$ сходятся к $f$ в указанных топологиях $S$. Для этого достаточно установить сходимость к нулю функционала $d\left(f_{n}, g_{n}\right)$. Имеем

$$
\begin{aligned}
d\left(f_{n}, g_{n}\right)= & \int_{X^{2}}\left|f_{n}(t)-g_{n}(t)\right|\left|f_{n}(s)-g_{n}(s)\right||m|(d t, d s) \\
= & \int_{X^{2}}\left|\sum_{k=1}^{n} \frac{M k}{n}\left(I_{B_{k}^{n}}(t)-I_{A_{k}^{n}}(t)\right)\right| \\
& \times\left|\sum_{k=1}^{n} \frac{M k}{n}\left(I_{B_{k}^{n}}(s)-I_{A_{k}^{n}}(s)\right)\right||m|(d t, d s)
\end{aligned}
$$




$$
\begin{aligned}
\leqslant & \int_{X_{2}}\left(\sum_{k=1}^{n} \frac{M k}{n} I_{\left(B_{k} \bar{A}_{k} \cup \bar{B}_{k} A_{k}\right)}(t)\right) \\
& \times\left(\sum_{k=1}^{n} \frac{M k}{n} I_{\left(B_{k} \bar{A}_{k} \cup \bar{B}_{k} A_{k}\right)}(s)\right)|m|(d t, d s) \\
= & \sum_{k, l=1}^{n} \frac{M^{2} k l}{n^{2}} \int I_{\left(B_{k} \bar{A}_{k} \cup \bar{B}_{k} A_{k}\right)}(t) I_{\left(B_{l} \bar{A}_{l} \cup \bar{B}_{l} A_{l}\right)}(s)|m|(d t, d s) \\
= & \sum_{k, l=1}^{n} \frac{M^{2} k l}{n^{2}}|m|\left(\left(B_{k} \bar{A}_{k} \cup \bar{B}_{k} A_{k}\right),\left(B_{l} \bar{A}_{l} \cup \bar{B}_{l} A_{l}\right)\right) \\
\leqslant & M^{2} \sum_{k=1}^{n}|m|\left(X, B_{k} \bar{A}_{k} \cup \bar{B}_{k} A_{k}\right) \leqslant M^{2} \sum_{k=1}^{n} \rho\left(B_{k}^{n}, A_{k}^{n}\right) \leqslant M^{2} n^{-1} \longrightarrow 0
\end{aligned}
$$

при $n \rightarrow \infty$. Таким образом, мы доказали существование последовательности ступенчатых функций вида

$$
f_{n}=\sum_{k=1}^{n} a_{k}^{n} I_{C_{k}^{n}}(t), \quad C_{k}^{n} \in \mathfrak{M},
$$

приближающих $f$ в двух введенных выше метриках $S$.

Пусть теперь $f$ неотрицательна и неограничена. Рассмотрим срезку функции $f$ на уровне $M: f^{M}(t)=f(t) I_{\{t: f(t)<M\}}(t)$. Тогда

$$
\begin{aligned}
& \int_{X^{2}}\left|f^{M}(t)-f(t)\right|\left|f^{M}(s)-f(s)\right||m|(d t, d s) \\
& =\int_{C(M)}\left|f^{M}(t)-f(t)\right|\left|f^{M}(s)-f(s)\right||m|(d t, d s),
\end{aligned}
$$

где $C(M)=\left\{(t, s) \in X^{2}: \min (f(t), f(s)) \geqslant M\right\}$. Но тогда правая часть последнего равенства сходится к нулю при $M \rightarrow \infty$ в силу теоремы РадонаНикодима и того обстоятельства, что $|m|(C(M)) \rightarrow 0$ при $M \rightarrow \infty$ (напомним, что $|m|$ есть $\sigma$-аддитивная мера, которая, стало быть, удовлетворяет теореме непрерывности для любой подпоследовательности сужающихся множеств). Таким образом, $f^{M} \rightarrow f$ в любой из двух рассматриваемых топологий в $S$, и построение соответствующих ступенчатых функций сводится к уже разобранному случаю ограниченных ядер. Наконец, от знакопостоянства функции $f$ можно отказаться, представив ее в виде разности двух неотрицательных функций $f^{+}$и $f^{-}$.

Переходим к доказательству основного утверждения теоремы. Ввиду полноты пространства $\mathscr{L}_{2}$ нам достаточно проверить критерий Коши, т.е. фундаментальность последовательности случайных величин $\left\{\eta_{n} \equiv \eta\left(f_{n}\right)\right\}$ в норме $\mathscr{L}_{2}$. Из (5) получаем

$$
\begin{aligned}
\mathbf{E}\left(\eta_{l}-\eta_{n}\right)^{2} & =\int_{X^{2}}\left(f_{n}(t)-f_{l}(t)\right)\left(f_{n}(s)-f_{l}(s)\right) m(d t, d s) \\
& =\left\|f_{n}-f_{l}\right\|^{2} \leqslant d_{0}\left(f_{n}-f_{l}\right)^{2} \leqslant\left\|f_{n}-f_{l}\right\|_{\mathscr{L}_{2}}^{2} .
\end{aligned}
$$


В силу неравенства треугольника правая часть последнего неравенства стремится к нулю при $n, l \rightarrow \infty$ в любом из трех приведенных случаев.

Таким образом, фундаментальность $\left\{f_{n}\right\}$ в одной из трех указанных норм влечет за собой фундаментальность в $\mathscr{L}_{2}$ последовательности $\left\{\eta_{n}\right\}$. В силу полноты пространства $\mathscr{L}_{2}$ последовательность $\left\{\eta_{n}\right\}$ сходится в среднеквадратичном к некоторой случайной величине $\eta(f)$. Независимость этого предела от последовательности $\left\{f_{n}\right\}$ легко доказывается от противного с использованием вышеприведенного неравенства. Теорема доказана.

О п р е д е л е н и е 3 . Назовем предельную случайную величину $\eta(f) \equiv \int f(t) \mu(d t)$ из теоремы 1 стохастическим интегралом функции $f$ по мере $\mu$.

Этот стохастический интеграл определен корректно для любой функции $f$, лежащей в одном из трех упомянутых выше линейных нормированных пространств, и представляет собой непрерывный линейный оператор, отображающий в $\mathscr{L}_{2}$ одно из этих трех пространств. При этом $\overline{\mathscr{L}}_{2}$ - гильбертово пространство, а $\left(S, d_{0}\right)$ будет банаховым, скажем, в условиях предложения 3 .

Отметим, что конструкция кратного стохастического интеграла

$$
\int f\left(x_{1}, \ldots, x_{k}\right) \mu\left(d x_{1}\right) \cdots \mu\left(d x_{k}\right)
$$

принципиально ничем не отличается от приведенной выше схемы. В самом деле, декартова степень $\mathfrak{M}^{k}:=\mathfrak{M} \times \cdots \times \mathfrak{M}$ будет полукольцом в $X^{k}$ с единицей. Множества из $\mathfrak{M}^{k}$ представляют собой канонические параллелепипеды в $X^{k}$. Соответствуюшая стохастическая мера на $\mathfrak{M}^{k}$ задается произведением

$$
\tilde{\mu}\left(A_{1} \times \cdots \times A_{k}\right):=\mu\left(A_{1}\right) \times \cdots \times \mu\left(A_{k}\right),
$$

где $A_{j} \in \mathfrak{M}$ и $\mu$ - элементарная стохастическая мера на $\mathfrak{M}$. Наконец, ковариационная мера на прямом произведении упомянутых параллелепипедов определяется по формуле

$$
\begin{aligned}
m(A \times B) & \equiv m\left(A_{1} \times \cdots \times A_{k} \times B_{1} \times \cdots \times B_{k}\right) \\
& =\mathbf{E} \mu\left(A_{1}\right) \cdots \mu\left(A_{k}\right) \mu\left(B_{1}\right) \cdots \mu\left(B_{k}\right),
\end{aligned}
$$

где $A=A_{1} \times \cdots \times A_{k}, B=B_{1} \times \cdots \times B_{k}, A_{j}, B_{j} \in \mathfrak{M}$. Так что в приведенной выше схеме построения меняется только «размерность» пространства $X$ и стохастическая мера имеет приведенную выше специальную структуру, в силу чего доказательство принадлежности ядра $f$ к тому или иному пространству для конкретных стохастических мер $\mu$, как правило, будет более сложным по сравнению с «одномерной» конструкцией (см. раздел 3). 
Важно отметить, что мера $\widetilde{\mu}$, вообще говоря, уже не будет ортогональной, даже если образуюшая ее мера $\mu$ ортогональна. Например, пусть

$$
\widetilde{\mu}\left(A_{1} \times \cdots \times A_{k}\right)=W_{\lambda}\left(A_{1}\right) \cdots W_{\lambda}\left(A_{k}\right),
$$

где $k=2 n$ - четное. Положим $A=A_{1}^{k}, B=B_{1}^{k}$, причем $A_{1} \cap B_{1}=\varnothing$ (стало быть, $A \cap B=\varnothing$ ). Тогда легко видеть, что

$$
m(A \times B)=\mathbf{E} W_{\lambda}\left(A_{1}\right)^{2 n} \mathbf{E} W_{\lambda}\left(B_{1}\right)^{2 n}=((2 n-1) ! !)^{2} \lambda\left(A_{1}\right)^{n} \lambda\left(B_{1}\right)^{n},
$$

т.е. условие ортогональности (M2) для меры $\widetilde{\mu}$ не выполнено.

3. Примеры. В этом разделе будет обсуждаться вопрос проверки условий теоремы 1 . Другими словами, нам надо указать легко проверяемые достаточные условия принадлежности функции $f$ пространству $S$, построенному по ковариационной мере той или иной элементарной стохастической меры.

Рассмотрим центрированный случайный процесс $\xi(t)$, заданный на отрезке $[0, T]$ и имеющий ковариационную функцию $\Phi(t, s):=\mathbf{E} \xi(t) \xi(s)$, где $t, s \in X \equiv[0, T]$. Рассмотрим следуюшее полукольцо с единицей подмножеств отрезка $[0, T]$ :

$$
\mathfrak{M}=\{(t, t+\delta] ; 0<t<t+\delta \leqslant T\} \bigcup\{[0, \delta] ; 0<\delta \leqslant T\} .
$$

Любой такой случайный процесс индуцирует элементарную стохастическую меру на этом полукольце:

$$
\mu((t, t+\delta]):=\xi(t+\delta)-\xi(t),
$$

где при $t=0$ по этой формуле определяется мера замкнутого интервала $[0, \delta]$ (в дальнейшем это обстоятельство больше не оговаривается). Тогда соответствующая ковариационная мера $m$ канонического прямоугольника в $X^{2}$ определяется по формуле

$$
\begin{aligned}
& m\left(\left(t, t+\delta_{1}\right] \times\left(s, s+\delta_{2}\right]\right) \\
& \quad=\mathbf{E}\left(\xi\left(t+\delta_{1}\right) \xi\left(s+\delta_{2}\right)-\xi(t) \xi\left(s+\delta_{2}\right)-\xi\left(t+\delta_{1}\right) \xi(s)+\xi(t) \xi(s)\right) \\
& \quad=\Delta_{\delta_{1}, \delta_{2}}(t, s),
\end{aligned}
$$

где $\Delta_{\delta_{1}, \delta_{2}}(t, s):=\Phi\left(t+\delta_{1}, s+\delta_{2}\right)+\Phi(t, s)-\Phi\left(t+\delta_{1}, s\right)-\Phi\left(t, s+\delta_{2}\right)-$ так называемая двойная разность функции $\Phi(t, s)$. Будем говорить, что функция $\Phi(t, s)$ имеет ограниченную вариачию по совокупности переменныз , если найдется такая постоянная $C$, что каковы бы ни были натуральные числа $n, l$ и разбиение множества $[0, T]^{2}$ на прямоугольники вида

$$
\begin{gathered}
\left(t_{i}, t_{i+1}\right] \times\left(s_{j}, s_{j+1}\right], \\
0=t_{0}<t_{1}<\cdots<t_{n}=T, \quad 0=s_{0}<s_{1}<\cdots<s_{l}=T
\end{gathered}
$$


(при этом при $i=0$ или $j=0$ соответствующие элементы разбиения полагаются замкнутыми «слева»), будет выполнено неравенство

$$
\sum_{i, j}\left|\Delta_{\delta_{1 i}, \delta_{2 j}}\left(t_{i}, s_{j}\right)\right| \leqslant C
$$

где $\delta_{1 i}=t_{i+1}-t_{i}, \delta_{2 j}=s_{j+1}-s_{j}$.

Предложение 4. Если ковариачионная функция $\Phi(t, s)$ имеет ограниченную вариацию по совокупности переменных, то мера $m$ является $\sigma$-аддитивной знакопеременной мерой на б-алгебре борелевских подмножеств квадрата $[0, T]^{2}$.

Д о к а з а т е л ь с т в о. Чтобы доказать $\sigma$-аддитивность меры $m$ на $[0, T]^{2}$, достаточно установить ее $\sigma$-аддитивность на полукольце полуоткрытых канонических прямоугольников в $[0, T]^{2}$ (см., например, [12]).

Пусть $A_{i}=\left(x_{i}, x_{i+1}\right], B_{j}=\left(y_{j}, y_{j+1}\right], i, j=0,1,2, \ldots$ Пусть, далее, $A=\bigcup A_{i}=\left(x_{0}, x_{\infty}\right], B=\bigcup B_{i}=\left(y_{0}, y_{\infty}\right]$, где $x_{\infty}=\lim _{i} x_{i}, y_{\infty}=\lim _{j} y_{j}$. Ряд $\sum_{i, j} m\left(A_{i} \times B_{j}\right)$ абсолютно сходится в силу определения меры $m$ и того обстоятельства, что $\Phi$ - функция ограниченной вариации по совокупности переменных. Тогда, применяя теорему Фубини, имеем

$$
\begin{aligned}
\sum_{i, j=0}^{\infty} m\left(A_{i} \times B_{j}\right) & =\sum_{i} \sum_{j} \Delta_{\delta_{1 i}, \delta_{2 j}}\left(t_{i}, s_{j}\right) \\
& =\sum_{i}\left(\Phi\left(t_{i+1}, s_{\infty}\right)+\Phi\left(t_{i}, s_{0}\right)-\Phi\left(t_{i+1}, s_{0}\right)-\Phi\left(t_{i}, s_{\infty}\right)\right) \\
& =\Phi\left(t_{\infty}, s_{\infty}\right)+\Phi\left(t_{0}, s_{0}\right)-\Phi\left(t_{\infty}, s_{0}\right)-\Phi\left(t_{0}, s_{\infty}\right) \\
& =m(A \times B) .
\end{aligned}
$$

Предложение доказано.

Отметим, что при построении стохастических интегралов в [6] и [7] также рассматривались только ковариационные функции ограниченной вариации.

3.1. Процессы с регулярной ковариационной функцией. Мы начнем описание достаточных условий существования определенного выше стохастического интеграла с простейшего случая, когда двойная разность ковариационной функции процесса $\{\xi(t) ; t \in[0, T]\}$ допускает представление

$$
\Delta_{\delta_{1 i}, \delta_{2 j}} \Phi\left(t_{i}, s_{j}\right)=\int_{t_{i}}^{t_{i+1}} \int_{s_{j}}^{s_{j+1}} q(t, s) \lambda(d t) \lambda(d s),
$$

где $\lambda$ - произвольная конечная мера на $[0, T]$, функция $q(t, s)$ интегрируема на $[0, T]^{2}$ по указанной продакт-мере, а $t_{i}<t_{i+1}$ и $s_{j}<s_{j+1}$ произвольные упорядоченные пары чисел из $[0, T]$. Очевидно, в этом случае ковариационная функция $\Phi(t, s)$ имеет ограниченную вариацию. 
Как уже было показано, ковариационная мера $m$ произвольного канонического прямоугольника $\left(t_{i}, t_{i+1}\right] \times\left(s_{j}, s_{j+1}\right]$ вычисляется по формуле

$$
m\left(\left(t_{i}, t_{i+1}\right] \times\left(s_{j}, s_{j+1}\right]\right)=\Delta_{\delta_{1 i}, \delta_{2 j}} \Phi\left(t_{i}, s_{j}\right),
$$

откуда и следует соотношение $\frac{d|m|}{d \lambda_{0}}(t, s)=|q(t, s)|$, где $\lambda_{0}(d t \times d s)=$ $\lambda(d t) \lambda(d s), \quad$ и принадлежность функции $f$ пространствам $\mathscr{L}_{2}(\mu)$ или $\left(S, d_{0}\right)$ выражается соотношением

$$
\int_{X}|f(t) f(s)||q(t, s)| \lambda(d t) \lambda(d s)<\infty
$$

Кроме того, проекция полной вариации ковариационной меры будет равна

$$
\bar{m}(A)=\int_{A}\left(\int_{X}|q(t, s)| \lambda(d s)\right) \lambda(d t) .
$$

Обозначим $\tilde{q}(t):=\int_{X}|q(t, s)| \lambda(d s)$. Тогда принадлежность функции $f$ пространству $\overline{\mathscr{L}}_{2}$ следует из квадратичной суммируемости этой функции по мере $\widetilde{q}(t) \lambda(d t)$. Если же $\widetilde{q}(t)$ равномерно ограничена на отрезке $[0, T]$, то для построения стохастического интеграла, очевидно, достаточно проверить лишь квадратичную суммируемость $f$ на $[0, T]$ по мере $\lambda$ (скажем, по мере Лебега или считающей мере). Однако надо иметь в виду, что последние условия являются, вообще говоря, более ограничительными, чем требование существования вышеприведенного двойного интеграла. Например, в случае, когда плотность $q(t, s)$ ограничена, для корректного задания стохастического интеграла вместо квадратичной достаточно потребовать просто интегрируемость по мере $\lambda$ функции $f$ на $[0, T]$.

Рассмотрим введенный в предыдущем разделе кратный стохастический интеграл

$$
\int f\left(x_{1}, \ldots, x_{k}\right) \mu\left(d x_{1}\right) \cdots \mu\left(d x_{k}\right),
$$

который, как было отмечено, можно трактовать как описанный выше «одномерный» стохастический интеграл по элементарной стохастической мере $\widetilde{\mu}$ на полукольце $\mathfrak{M}^{k}$. Множества из $\mathfrak{M}^{k}$ представляют собой канонические параллелепипеды в $X^{k}$, и ковариационная мера на $X^{2 k}$ определяегся по формуле

$$
m(A \times B)=\mathbf{E} \tilde{\mu}(A) \tilde{\mu}(B)=\mathbf{E} \mu\left(A_{1}\right) \cdots \mu\left(A_{k}\right) \mu\left(B_{1}\right) \cdots \mu\left(B_{k}\right),
$$

где $A=A_{1} \times \cdots \times A_{k}, B=B_{1} \times \cdots \times B_{k}, A_{j}, B_{j} \in \mathfrak{M} ;$ при этом $\mu((t, t+\delta])=$ $\xi(t+\delta)-\xi(t)$.

В дальнейшем для вычисления ковариационной меры $m$ в случае кратных интегралов мы будем предполагать, что $\xi(t)$ - чентрированный гауссовский прочесс. Тогда любые конечные наборы случайных 
величин вида $\mu((t, t+\Delta])$ будут иметь совместное гауссовское распределение.

Посчитаем меру $m\left(A_{1} \times \cdots \times A_{2 k}\right) 2 k$-мерного куба, где $A_{i}=$ $\left(t_{i}, t_{i}+\delta\right]$. Воспользуемся известной формулой для подсчета смешанных четных моментов гауссовских векторов (см., например, [11]). Тогда

$$
m\left(A_{1} \times \cdots \times A_{2 k}\right)=\mathbf{E} \mu\left(A_{1}\right) \cdots \mu\left(A_{2 k}\right)=\sum \prod \Delta_{\delta, \delta} \Phi\left(t_{i}, t_{j}\right) .
$$

Здесь и далее, если не оговорено противное, сумма берется по всевозможным разбиениям множества индексов $\{1, \ldots, 2 k\}$ на пары, а произведение - по всем таким парам в фиксированном разбиении. В условиях и обозначениях примера из начала этого пункта имеем

$$
m\left(A_{1} \times \cdots \times A_{2 k}\right)=\int_{A_{1}} \cdots \int_{A_{2 k}} \bar{q}\left(t_{1}, \ldots, t_{2 k}\right) \lambda\left(d t_{1}\right) \cdots \lambda\left(d t_{2 k}\right),
$$

где

$$
\bar{q}\left(t_{1}, \ldots, t_{2 k}\right)=\sum \prod q\left(t_{i}, t_{j}\right),
$$

т.е. мера $|m|$ имеет плотность $|\bar{q}|$ относительно соответствующей $2 k$ мерной продакт-меры. Так что принадлежность $f\left(t_{1}, \ldots, t_{k}\right)$ к пространствам $\mathscr{L}_{2}(\widetilde{\mu})$ или $\left(S, d_{0}\right)$ обеспечивается условием

$$
\int\left|f\left(t_{1}, \ldots, t_{k}\right) f\left(t_{k+1}, \ldots, t_{2 k}\right) \bar{q}\left(t_{1}, \ldots, t_{2 k}\right)\right| \lambda\left(d t_{1}\right) \cdots \lambda\left(d t_{2 k}\right)<\infty .
$$

В случае, когда $\lambda$ - мера Лебега на прямой и одномерная проекция стохастической продакт-меры порождена приращениями регулярного фрактального броуновского движения (см. п. 3.4), аналогичное условие существования кратного стохастического интеграла приведено в [8]. В этом случае в условие (7) нужно подставить функцию

$$
q(t, s)= \begin{cases}h(2 h-1)|t-s|^{2 h-2}, & t \neq s \\ 0, & t=s\end{cases}
$$

где $h \in\left(\frac{1}{2}, 1\right)$.

3.2. Процессы с факторизующейся ковариационной функцией. Приведенные в предыдущем пункте процессы с абсолютно непрерывными (относительно соответствующих продакт-мер) ковариационными функциями образуют довольно узкий класс, и их нельзя назвать типичными для приложений теории случайных процессов. Одним из свойств абсолютно непрерывных ковариационных мер является их равенство нулю на любых диагональных подпространствах, если только компонента $\lambda$ упомянутой продакт-меры не имеет атомов. Однако далеко не всегда ковариационная мера обладает указанным свойством. В качестве иллюстрации сказанному рассмотрим следующий класс случайных процессов. 
Пусть ковариационная функция интегрирующего процесса $\{\xi(t)$; $t \in[0, T]\}$ допускает представление

$$
\Phi(t, s)=G(\min (t, s)) H(\max (t, s))
$$

где неотрицательные компоненты факторизации $G$ и $H$ таковы, что отношение $G / H$ не убывает и не обращается в нуль на открытом интервале $(0, T)$. В [15] установлено, что функция в (8) будет положительно определенной (т.е. ковариационной) в классическом смысле. В частности, это представление имеет место для любых невырожденных на $(0, T)$ гауссовских марковских процессов (см. [15]). Например, для броуновского моста на $[0,1]$ справедливо представление (8) с компонентами $G(z)=z$ и $H(z)=1-z$, а для стандартного винеровского процесса на $[0, \infty)$ имеем $G(z)=z$ и $H(z) \equiv 1$. Для произвольного стационарного гауссовского марковского процесса на $[0, \infty)$ компоненты факторизации в (8) вычисляются по формулам $G(z)=\exp (\alpha z)$ и $H(z)=\exp (-\alpha z)$, где $\alpha>0$.

Для простоты дальнейшего анализа предположим дополнительно, что обе компоненты факторизации в (8) равномерно ограничены и абсолютно непрерывны относительно меры Лебега на $[0, T]$, при этом $G$ не убывает, а $H$ не возрастает. Покажем, что мера $m$ произвольного внедиагонального прямоугольника отрицательна. В самом деле, если $s<s+\delta \leqslant t<t+\Delta$, то

$$
\begin{aligned}
& m((s, s+\delta] \times(t, t+\Delta]) \\
& \quad=G(s) H(t)+G(s+\delta) H(t+\Delta)-G(s) H(t+\Delta)-G(s+\delta) H(t) \\
& \quad=(G(s+\delta)-G(s))(H(t+\Delta)-H(t)) \leqslant 0 .
\end{aligned}
$$

Из (9) следует, что множество отрицательности меры $m$ представляет собой весь квадрат $[0, T]^{2}$ за вычетом диагонали и мера $m^{-}$конечна и абсолютно непрерывна относительно меры Лебега $\Lambda$ на плоскости; при этом

$$
\frac{d m^{-}}{d \Lambda}(t, s)=-G^{\prime}(t) H^{\prime}(s),
$$

где $G^{\prime}$ и $H^{\prime}$ - соответствующие производные Радона-Никодима функций $G$ и $H$.

Заметим теперь, что диагональное множество $\{(t, s): t=s\}$ всегда включено в множество положительности любой ковариационной меры. В самом деле, для любых $t, t+\Delta \in[0, T]$ имеем

$$
m((t, t+\Delta] \times(t, t+\Delta])=\mathbf{D}(\xi(t+\Delta)-\xi(t)) \geqslant 0 .
$$

Далее, для измельчаюшегося разбиения $\left\{t_{k}^{n}\right\}$ произвольного отрезка $(a, b] \subseteq[0, T]: a=t_{0}^{n}<\cdots<t_{m(n)}^{n}=b$, где $t_{k}^{n}=a+k(b-a) 2^{-n}$ и $m(n)=2^{n}$, 
объединения квадратов $\bigcup_{i \leqslant n}\left\{\left(t_{i-1}^{n}, t_{i}^{n}\right] \times\left(t_{i-1}^{n}, t_{i}^{n}\right]\right\}$, «нанизанных» на диагональ, по $n$ образуют последовательность вложенных подмножеств, пересечение которых совпадает с частью диагонали, заключенной внутри квадрата $(a, b] \times(a, b] ;$ причем в силу сделанного выше замечания мера $m$ каждого из указанных подмножеств неотрицательна. По свойству непрерывности конечных $\sigma$-аддитивных зарядов получаем, что ковариационная мера указанной части диагонали всегда неотрицательна. Легко видеть, что отсюда следует наше утверждение.

Таким образом, в рассматриваемом случае мера $m^{+}$сосредоточена на диагонали. Для того чтобы понять, как вычислять $m^{+}$, сначала рассмотрим $m((s, s+h] \times(s, s+h])$ при малых $h>0$. Имеем

$$
\begin{aligned}
m & ((s, s+h] \times(s, s+h]) \\
& =H(s+h)(G(s+h)-G(s))-G(s)(H(s+h)-H(s)) \\
& =\int_{s .}^{s+h}\left(H(z) G^{\prime}(z)-G(z) H^{\prime}(z)\right) d z+o(h) .
\end{aligned}
$$

Так что меру $m^{+}$квадрата $(s, s+\delta] \times(s, s+\delta$ ] (или, что то же, меру части диагонали, заключенной между точками $(s, s)$ и $(s+\delta, s+\delta)$ можно вычислить интегрированием на отрезке $[s, s+\delta]$ полученной одномерной плотности (см. вышеприведенное замечание о вычислении меры диагонали):

$$
m^{+}((s, s+\delta] \times(s, s+\delta])=\int_{s}^{s+\delta}\left(H(z) G^{\prime}(z)-G(z) H^{\prime}(z)\right) d z .
$$

Таким образом, положительная часть ковариационной меры также конечна и абсолютно непрерывна относительно одномерной меры Лебега, индуцированной на диагонали, т.е. в данном примере меры $m^{+}$и $m^{-}$ сингулярньц!

Понятно, что мера $m^{+}$любого прямоугольника $(a, b] \times(c, d]$ совпадет с величиной меры в (10) при условии, что $(s, s+\delta] \times(s, s+\delta]-$ максимальный по размеру диагональный квадрат, содержащийся в прямоугольнике $(a, b] \times(c, d]$.

Теперь, после сделанного замечания, с помощью (9) и (10) можем вычислить меру $|m|$ произвольного конечного прямоугольника, имеющего непустое пересечение с диагональным множеством:

$$
\begin{aligned}
& |m|((s, s+\delta] \times(t, t+\Delta]) \\
& =m^{+}((s, s+\delta],(t, t+\Delta])+m^{-}((s, s+\delta],(t, t+\Delta]) \\
& =\int_{s_{1}}^{s_{2}}\left(H(z) G^{\prime}(z)-G(z) H^{\prime}(z)\right) d z \\
& \quad+(G(s+\delta)-G(s))(H(t)-H(t+\Delta)),
\end{aligned}
$$


где $s_{1} \leqslant s_{2}$ - проекции на ось $s$ концов отрезка диагонали, заключенного внутри прямоугольника $(s, s+\delta] \times(t, t+\Delta]$. Отсюда легко получаем представление и для соответствующей проекции меры $|m|$ :

$$
\begin{aligned}
\bar{m}((s, s+\delta])= & \int_{s}^{s+\delta}\left(H(z) G^{\prime}(z)-G(z) H^{\prime}(z)\right) d z \\
& +(G(s+\delta)-G(s))(H(0)-H(T)) .
\end{aligned}
$$

Следовательно, плотность меры $\bar{m}$ на прямой вычисляется по формуле

$$
\frac{d \bar{m}}{d s}(s)=(H(s)+H(0)-H(T)) G^{\prime}(s)-G(s) H^{\prime}(s) .
$$

Таким образом, в данном примере при сделанных предположениях относительно функций $G$ и $H$ квадратичная интегрируемость функции $f$ по мере $\bar{m}$ означает, что

$$
\int_{0}^{T} f^{2}(s)\left(G^{\prime}(s)-G(s) H^{\prime}(s)\right) d s<\infty .
$$

Если же функции $G^{\prime}$ и $H^{\prime}$ равномерно ограничены на $[0, T]$, то упомянутая квадратичная интегрируемость следует из соответствующей интегрируемости по мере Лебега. Заметим, что в случае броуновского моста и в случае стационарного гауссовского марковского процесса (на конечном отрезке) функции $G$ и $H$ удовлетворяют этим требованиям.

Нетрудно также видеть, что конечность функционала $d_{0}$, вычисленного по мере $|m|$ в (11), эквивалентна условию (12). Иначе говоря, в данном примере пространства $\left(S, d_{0}\right)$ и $\overline{\mathscr{L}}_{2}$ совпадают, что качественно отличает его от соответствующего примера предыдущего пункта.

Понятно, что в вышеприведенных рассуждениях компоненты $G$ и $H$ могут быть абсолютно непрерывными относительно другой $\sigma$-конечной меры (например, считаюшей). Скажем, можно предложить ковариационную функцию с разрывными кусочно-постоянными факторизационными компонентами. Тогда мера $\bar{m}$ будет сосредоточена в не более чем счетном числе атомов, и условие принадлежности той или иной функции пространству $\overline{\mathscr{L}}_{2}$ будет описываться сходимостью некоторого ряда.

Теперь опять рассмотрим кратный интеграл, но уже построенный по центрированному гауссовскому марковскому процессу с теми же условиями на компоненты $G$ и $H$, что и в предыдушем примере, где для простоты рассуждений вместо абсолютной непрерывности предполагаем непрерывную дифференцируемость этих компонент. Аналогично примеру из предыдущего пункта имеем

$$
m\left(A_{1} \times \cdots \times A_{2 k}\right)=\sum \prod \Delta_{\delta, \delta} \Phi\left(t_{i}, t_{j}\right) .
$$

Будем считать длину ребра куба $\delta$ инфинитезимальной (т.е. «достаточно малой»): так что для любых $u<v$ имеет место неравенство 
$u+\delta<v-\delta$. Реализуется одна из двух ситуаций (см. предыдущий пример):

1) при $t_{i}=t_{j}$ выполнено

$$
\Delta_{\delta, \delta} \Phi\left(t_{i}, t_{j}\right)=\delta\left(H\left(t_{i}\right) G^{\prime}\left(t_{i}\right)-G\left(t_{i}\right) H^{\prime}\left(t_{i}\right)\right)+O\left(\delta^{2}\right) ;
$$

2) если $t_{i} \neq t_{j}$ (см. (9)), то

$$
\Delta_{\delta, \delta} \Phi\left(t_{i}, t_{j}\right)=\delta^{2} G^{\prime}\left(\min \left(t_{i}, t_{j}\right)\right) H^{\prime}\left(\max \left(t_{i}, t_{j}\right)\right)+o\left(\delta^{2}\right) .
$$

Обозначим

$$
\begin{aligned}
g_{1}(t) & :=H(t) G^{\prime}(t)-G(t) H^{\prime}(t), \\
g_{2}\left(t_{i}, t_{j}\right) & :=G^{\prime}\left(\min \left(t_{i}, t_{j}\right)\right) H^{\prime}\left(\max \left(t_{i}, t_{j}\right)\right) .
\end{aligned}
$$

Отметим, что в условиях предыдушего примера $g_{1}(t) \geqslant 0$ и $g_{2}\left(t_{i}, t_{j}\right) \leqslant 0$.

Будем называть $L$ диагональным подпространством в $X^{2 k}$, если из принадлежности $\left(x_{1}, \ldots, x_{2 k}\right) \in L$ следует равенство координат $x_{i}$ для тех или иных ( сло свободных переменных, задающих то или иное диагональное подпространство (или, иначе говоря, размерность этого подпространства), меняется от 1 (главная диагональ $\left\{\left(x_{1}, \ldots, x_{2 k}\right) \in X^{2 k}: x_{1}=\cdots=x_{2 k}\right\}$ ) до $2 k-1$ (например, гиперплоскость $\left\{\left(x_{1}, \ldots, x_{2 k}\right) \in X^{2 k}: x_{1}=x_{2}\right\}$ ). При этом размер каждой из таких групп индексов мы будем отождествлять с кратностью соответствующей переменной. Дополнение к объединению всех диагональных подпространств будем называть главныл подпространством $X^{2 k}$.

Предложение 5. Ковариационная мера $m$ может иметь ненулевую массу только на главном подпространстве и диагональных подпространствах, определяемьх переменными кратности не больше 2.

Д ок аз а тельст в о. Как уже было отмечено, величина $\Delta_{\delta, \delta} \Phi\left(t_{i}, t_{j}\right)$ при $t_{i}=t_{j}$ (скажем, если двойная разность построена по переменной кратности 2) имеет порядок малости $\delta$ в произведении вида $\prod \Delta_{\delta, \delta} \Phi\left(t_{i}, t_{j}\right)$. В противном случае $\Delta_{\delta, \delta} \Phi\left(t_{i}, t_{j}\right)=O\left(\delta^{2}\right)$. Пусть сначала все $2 k$ переменных в задании «минимальной» вершины $2 k$-мерного куба $A_{1} \times \cdots \times A_{2 k}$ попарно различны, т.е. мы рассматриваем точки главного подпространства. Тогда каждое из произведений в (13) имеет порядок малости $\delta^{2 k}$. Стало быть, сужение ковариационной меры $m$ на главное подпространство будет абсолютно непрерывным относительно $2 k$ мерной меры Лебега. Соответствуюшая производная Радона-Никодима (плотность) равна

$$
\sum \prod g_{2}\left(t_{i}, t_{j}\right)
$$

где сумма и произведения определены в (13). При этом, как легко видеть, эта производная будет неотрицательна или неположительна соответственно при четном или нечетном $k$. 
Далее, пусть одна из переменных, определяющих диагональное подпространство $L$, имеет кратность $l \geqslant 2$, а остальные - кратность 1 . Тогда нетрудно видеть, что минимальный порядок малости отдельного произведения в $(13)$ будет $\delta^{r(l)}$, где $r(l):=[l / 2]+2(k-[l / 2])$, a [·] целая часть числа. Причем этот порядок достигается на произведении в сумме (13), в котором максимальное число (т.е. $[l / 2])$ двойных разностей имеют одинаковые аргументы. Например, если $L=\left\{\left(t_{1}, t_{2}, t_{3}, t_{4}\right)\right.$ : $\left.t_{1}=t_{2}\right\}$, то главным (т.е. имеющим минимальный порядок малости) в (13) будет слагаемое, содержащее $\Delta_{\delta, \delta} \Phi\left(t_{1}, t_{2}\right) \Delta_{\delta, \delta} \Phi\left(t_{3}, t_{4}\right)$, так как оно даст порядок $\delta^{3}$ (здесь число свободных переменных равно трем). Слагаемое же, содержащее, например, $\Delta_{\delta, \delta} \Phi\left(t_{1}, t_{3}\right) \Delta_{\delta, \delta} \Phi\left(t_{2}, t_{4}\right)$, даст порядок малости $\delta^{4}$.

Кроме того, размерность $L$ будет равна величине $2 k-l+1$. Очевидно, для любых натуральных $l$ и $k(l \leqslant 2 k)$

$$
r(l)=2 k-\left[\frac{l}{2}\right] \geqslant 2 k-l+1
$$

при этом равенство здесь достигается только при $l=2$, и сужение меры $m$ на $L$ будет абсолютно непрерывным относительно меры Лебега в $\mathbf{R}^{2 k-l+1}$ с, вообще говоря, ненулевой плотностью

$$
g_{1}\left(t_{i_{0}}\right) \sum \prod_{(i, j) \in I_{0}} g_{2}\left(t_{i}, t_{j}\right)
$$

где $i_{0}$ удовлетворяет соотношению $t_{i_{0}}=t_{j_{0}}$ для некоторого $j_{0}$, т.е. переменная $t_{i_{0}}$ в описании диагонального подпространства $L$ имеет кратность 2 , сумма берется по всем разбиениям на пары множества индексов $I_{0}:=\left\{i: i \leqslant 2 k, i \neq i_{0}, j_{0}\right\}$; здесь и всюду в дальнейшем условимся, что произведение по пустому множеству индексов (скажем, $I_{0}=\varnothing$ при $k=1$ ) равно 1.

Таким образом, в рассматриваемом случае мера $m$ может иметь ненулевую массу на $L$ только в случае, когда $l=2$ или $L-$ главное подпространство.

Пусть теперь диагональное подпространство $L$ определяется $n$ переменными кратности соответственно $l_{1}, \ldots, l_{n} \geqslant 2$ и оставшимися кратности 1. Аналогично предыдущему заключаем, что размерность $L$ равна $2 k-l_{1}-\cdots-l_{n}+n$, а минимальный возможный порядок малости отдельного произведения в (13) равен $\delta^{r}$, где

$$
r:=2 k-\left[\frac{l_{1}}{2}\right]-\cdots-\left[\frac{l_{n}}{2}\right] \geqslant 2 k-l_{1}-\cdots-l_{n}+n .
$$

При этом, как следует из только что проведенных рассуждений, последнее неравенство будет строгим, если $l_{i}>2$ хотя бы для одного $i \leqslant n$. В противном случае сужение ковариационной меры на $L$ будет абсолютно 
непрерывным относительно меры Лебега в $\mathbf{R}^{r}$ и соответствующая плотность будет равна

$$
\prod_{(l, d) \in I_{n}} g_{1}\left(t_{l}\right) \sum \prod_{(i, j) \in \bar{I}_{n}} g_{2}\left(t_{i}, t_{j}\right)
$$

где $I_{n} \equiv I_{n}(L)=\left\{\left(i_{k}, j_{k}\right) ; k \leqslant n\right\}$ - множество пар индексов переменных в описании диагонального подпространства $L$, имеющих кратность 2 (т.е. $t_{i_{k}}=t_{j_{k}}$ ), а $\bar{I}_{n}$ - дополнительное к $I_{n}$ множество пар индексов $(i, j)$ фиксированного разбиения; сумма берется по всем разбиениям на пары множества индексов $\left\{i \leqslant 2 k: i \neq i_{k}, j_{k}, k \leqslant n\right\}$.

Заметим, что число множителей в первом произведении будет равно числу свободных переменных кратности 2 , а число множителей во втором произведении будет равно половине числа переменных кратности 1 .

Стало быть, ковариационная мера произвольного $\sigma\left(\mathfrak{M}^{2 k}\right)$-измеримого множества $D \in X^{2 k}$ будет равна

$$
m(D)=\sum_{L} \int_{D \cap L} \prod_{l=1}^{n} g_{1}\left(s_{l}\right) \sum \prod_{(i, j) \in I_{2}} g_{2}\left(t_{i}, t_{j}\right) d s_{1} \cdots d s_{n} d t_{1} \cdots d t_{r-n}
$$

где внешняя сумма берется по главному и всем диагональным подпространствам $L$, которые задаются только переменными кратности 1 и 2; $r \equiv 2 k-n$ - размерность подпространства $L$, содержащего $n$ переменных $\left\{s_{i}\right\}$ кратности 2 и $2(k-n)$ переменных $\left\{t_{i}\right\}$ кратности 1 ; при $n=0$ интегрирование ведется по главному подпространству; внутренняя сумма берется по всем разбиениям на пары множества индексов $\{i: i \leqslant 2(k-n)\}$, а $I_{2}-$ множество таких пар в фиксированном разбиении. При $k=n$ в (14) по определению полагаем $\sum \prod \equiv 1$.

Для любой функции $f\left(t_{1}, \ldots, t_{k}\right)$ введем обозначение

$$
\varphi_{f}\left(t_{1}, \ldots, t_{2 k}\right):=f\left(t_{1}, \ldots, t_{k}\right) f\left(t_{k+1}, \ldots, t_{2 k}\right) .
$$

Из (14) следует, что для сушествования кратного стохастического интеграла от функции $f\left(t_{1}, \ldots, t_{k}\right)$ по заряду с ковариационной мерой $(14)$ достаточно, чтобы для любого $n=0,1, \ldots, k$ сушествовали, во-первых, интегралы

$$
\begin{aligned}
& \int\left|\varphi_{f}\left(s_{1}, s_{1}, s_{2}, s_{2}, \ldots, s_{n}, s_{n}, t_{1}, \ldots, t_{2 k-2 n}\right)\right| \\
& \quad \times \prod_{i=1}^{n} g_{1}\left(s_{i}\right) \sum \prod_{(i, j) \in I_{2}}\left|g_{2}\left(t_{i}, t_{j}\right)\right| d s_{1} \cdots d s_{n} d t_{1} \cdots d t_{2 k-2 n}
\end{aligned}
$$

а во-вторых, аналогичные интегралы при всех перестановках аргументов у функции $\varphi_{f}\left(s_{1}, s_{1}, s_{2}, s_{2}, \ldots, s_{n}, s_{n}, t_{1}, \ldots, t_{2 k-2 n}\right)$.

Отметим, что в важном частном случае, когда функции $g_{1}$ и $g_{2}$ равномерно ограничены, условия квадратичной интегрируемости (по мере 
Лебега) ядра $f\left(t_{1}, \ldots, t_{k}\right)$ на главном и всех диагональных подпространствах $X^{k}$, которые задаются переменными кратности 1 и 2 , являются достаточными для существования указанных выше интегралов, а стало быть, они обеспечат корректное задание соответствующего кратного стохастического интеграла. В частности, если продакт-мера построена по прирашениям стандартного винеровского процесса, то $g_{1} \equiv 1$ и $g_{2} \equiv 0$ и, как нетрудно видеть, упомянутую квадратичную интегрируемость достаточно проверить на диагональных подпространствах, определяемых только переменными кратности 2. Если же ограничиться рассмотрением ядер $f$, отличных от нуля лишь на главном подпространстве (как это сделано, например, в [5]), то условие построения соответствуюшего кратного стохастического интеграла становится совсем простым (cм. [5]):

$$
\int f\left(t_{1}, \ldots, t_{k}\right)^{2} d t_{1} \cdots d t_{k}<\infty
$$

3.3. Стохастические меры, связанные со статистическим анализом зависимых наблюдений. Рассмотрим еще одну модель стохастической меры, построенной по приращениям случайного процесса с ковариационной функцией вида

$$
\Phi(t, s)=\sum_{k} \Phi_{k}(t, s),
$$

где каждая из функций $\Phi_{k}(t, s)$ тоже ковариационная, имеющая, вообще говоря, несколько иную, чем в (8), структуру.

Пусть $\left\{\xi_{n}, n \geqslant 0\right\}$ - последовательность одинаково распределенных на $[0,1]$ стационарно связанных случайных величин с функцией распределения $F(t)$. Рассмотрим центрированный гауссовский процесс $Y(t)$ с ковариационной функцией

$$
\begin{aligned}
\Phi(t, s)= & \mathbf{E} Y(t) Y(s)=\mathbf{E} g_{s}\left(\xi_{0}\right) g_{t}\left(\xi_{0}\right) \\
& +\sum_{k \geqslant 1} \mathbf{E} g_{s}\left(\xi_{0}\right) g_{t}\left(\xi_{k}\right)+\sum_{k \geqslant 1} \mathbf{E} g_{s}\left(\xi_{k}\right) g_{t}\left(\xi_{0}\right),
\end{aligned}
$$

где $g_{t}(x)=I_{[0, t]}(x)-F(t)$, а $I_{A}(x)$ - индикаторная функция множества $A$; при этом предполагается, что ряды в правой части этого соотношения абсолютно сходятся для любых $t, s \in[0,1]$. Такие гауссовские процессы возникают как слабые пределы для последовательности стандартных эмпирических процессов, построенных по выборкам $\left\{\xi_{i} ; i \leqslant n\right\}$ при выполнении определенных условий зависимости элементов последовательности $\left\{\xi_{i}\right\}$ (см., например, [14]). Отметим, что для любого $k \geqslant 0$ имеет место очевидное представление

$$
\begin{aligned}
\mathbf{E} g_{s}\left(\xi_{0}\right) g_{t}\left(\xi_{k}\right) & =\int\left(I_{[0, s]}(x)-F(s)\right)\left(I_{[0, t]}(y)-F(t)\right) \mathbf{P}\left\{\xi_{0} \in d x, \xi_{k} \in d y\right\} \\
& =F_{\xi_{0}, \xi_{k}}(s, t)-F(t) F(s)
\end{aligned}
$$


где $F_{\xi_{0}, \xi_{k}}(s, t)$ - совместная функция распределения случайных величин $\xi_{0}$ и $\xi_{k}$; при этом лишь при $k=0$ соответствующая ковариационная функция имеет структуру (8), т.е. является факторизующейся. Благодаря именно этому обстоятельству структура ковариационной меры в данном примере качественно ничем не отличается от структуры ковариационной меры, построенной в п. 3.2 .

Пусть функция распределения $F(t)$ имеет плотность $F^{\prime}(t)$. Пусть, кроме того, совместные функции распределения пар $\left(\xi_{0}, \xi_{k}\right)$ при всех $k \geqslant 1$ также имеют соответствующие плотности $F_{\xi_{0}, \xi_{k}}^{\prime \prime}(s, t)$. Обозначим

$$
b(s, t):=\sum_{k \geqslant 1}\left(F_{\xi_{0} \xi_{k}}^{\prime \prime}(s, t)+F_{\xi_{0}, \xi_{k}}^{\prime \prime}(t, s)-2 F^{\prime}(t) F^{\prime}(s)\right) .
$$

При этом предполагается, что ряд из модулей элементов, стоящих под знаком суммы в приведенной формуле, сходится для почти всех $t, s \in[0,1]$ и представляет собой интегрируемую на $[0,1]^{2}$ функцию. Обозначим через $\mathscr{P}$ вероятностную меру на $[0,1]$, соответствующую функции распределения $F$. Тогда

$$
\begin{aligned}
m((t, t+\Delta] \times(s, s+\Delta])= & \mathscr{P}((t, t+\Delta] \cap(s, s+\Delta]) \\
& +\int_{t}^{t+\Delta} \int_{s}^{s+\Delta}\left[b(u, v)-F^{\prime}(u) F^{\prime}(v)\right] d u d v
\end{aligned}
$$

и мера $|m|$ вне диагонали будет иметь плотность

$$
\phi(s, t):=\left|b(s, t)-F^{\prime}(t) F^{\prime}(s)\right|
$$

относительно двумерной меры Лебега. Окончательно имеем

$$
d_{0}^{2}(f)=\int f^{2}(t) \mathscr{P}(d t)+\int|f(t)||f(s)| \phi(t, s) d t d s .
$$

Так что интегрируемость на $[0,1]^{2}$ функции $\phi(s, t)$ в степени $p>1$ и интегрируемость $f$ с квадратом по мере $\mathscr{P}$ определяет и соответствующий класс ядер $f$, который вкладывается в пространство $\left(S, d_{0}\right)$. В частности, если функция $\phi(s, t)$ равномерно ограничена на $[0,1]^{2}$, то для принадлежности $f \in\left(S, d_{0}\right)$ достаточно потребовать ее интегрируемость по Лебегу на $[0,1]$ (т.е. $\left.f \in L_{1}([0,1], d t)\right)$ и принадлежность пространству $L_{2}([0,1], \mathscr{P})$. Если же и плотность $F^{\prime}(\cdot)$ ограничена, то для существования соответствующего стохастического интеграла достаточно потребовать квадратичную интегрируемость функции $f$ на отрезке $[0,1]$.

Теперь рассмотрим кратный стохастический интеграл, построенный `по введенному выше гауссовскому процессу $\{Y(t) ; t \in[0,1]\}$. Для простоты рассуждений дополнительно предположим, что функции $F^{\prime}$ и $b(s, t)$ непрерывны. Исследуем двойную разность $\Delta_{\delta, \delta} \Phi\left(t_{i}, t_{j}\right)$ при инфинитезимальном $\delta$ (см. п. 3.2). Если $t_{i}=t_{j}$, то

$$
\Delta_{\delta, \delta} \Phi\left(t_{i}, t_{j}\right)=m\left(\left(t_{i}, t_{i}+\delta\right]^{2}\right)=\mathscr{P}\left(\left(t_{i}, t_{i}+\delta\right]\right)+O\left(\delta^{2}\right)=F^{\prime}\left(t_{i}\right) \delta+o(\delta) .
$$


Если же $t_{i} \neq t_{j}$, то

$$
\Delta_{\delta, \delta} \Phi\left(t_{i}, t_{j}\right)=\phi\left(t_{i}, t_{j}\right) \delta^{2}+o\left(\delta^{2}\right)
$$

так как интервалы $\left(t_{i}, t_{i}+\delta\right]$ и $\left(t_{j}, t_{j}+\delta\right]$ не пересекаются в силу инфинитезимальности $\delta$. Таким образом, данная конструкция полностью повторяет схему построения кратного интеграла из п. 3.2 при $g_{1}(t)=F^{\prime}(t)$ и $g_{2}(t, s)=\phi(t, s)$.

3.4. Случай мажорируемой ковариационной меры. В этом пункте мы предложим достаточно простой способ проверки условий теоремы 1 для одного специального класса ковариационных функций.

Пусть мера $|m|$, заданная на $\sigma$-алгебре $\sigma\left(\mathfrak{M}^{2}\right)$ декартова квадрата $X^{2}$ произвольного пространства $X$, удовлетворяет следуюшему условию мажорирования:

$$
|m|(d x, d y) \leqslant \phi(x, y) m_{0}(d x) m_{0}(d y),
$$

где $\phi \in L_{p}\left(X^{2}, m_{0}^{2}\right)$ для некоторого $p>1$, а $m_{0}$ - конечная мера на $\sigma(\mathfrak{M})$. Иными словами, ковариационная мера абсолютно непрерывна относительно продакт-меры $m_{0}^{2}$. Тогда, воспользовавшись классическим неравенством Гёльдера, имеем

$$
\begin{aligned}
d_{0}^{2}(f)= & \int_{X^{2}}|f(x)||f(y)||m|(d x, d y) \\
\leqslant & \int_{X^{2}}|f(x)||f(y)| \phi(x, y) m_{0}(d x) m_{0}(d y) \\
\leqslant & \left(\int_{X^{2}}|f(x) f(y)|^{p /(p-1)} m_{0}(d x) m_{0}(d y)\right)^{(p-1) / p} \\
& \times\left(\int_{X^{2}}(\phi(x, y))^{p} m_{0}(d x) m_{0}(d y)\right)^{1 / p}<\|f\|_{p /(p-1)}^{2}\|\phi\|_{p}<\infty,
\end{aligned}
$$

если $f \in L_{p /(p-1)}\left(X^{2}, m_{0}\right)$ (здесь и далее символами $\|\cdot\|_{q}$ обозначаются соответствующие $L_{q}$-нормы). Отметим, что при достаточно больших $p$ показатель $p /(p-1)$ может быть сколь угодно близким к 1 , а для ограниченных $\phi$ он в точности равен 1 .

Если функция $\phi$ имеет специальный вид, то можно предложить еще более точные, чем в $(15)$, оценки $d_{0}$-нормы функции $f$. Пусть $X=[0, T]$ и $m_{0}(d t)=d t-$ мера Лебега на прямой.

Предложение 6. Если $\phi(x, y)=\varphi(x-y)$, причем $\varphi \in L_{p}(X, d t) \partial л я$ некоторого $p \geqslant 1$, то пространство $L_{2 p /(2 p-1)}(X, d t)$ вложено в $S$.

Д ок а з а т л в с в о. Воспользуемся неравенством Юнга (см. [16]):

$$
\|J\|_{q} \leqslant\|\varphi\|_{p}\|f\|_{r}
$$

где $1 \leqslant r \leqslant q \leqslant \infty, 1-1 / r+1 / q=1 / p, f \in L_{r}(R, d t), \varphi \in L_{p}(R, d t)$ и $J(x)=\int f(y) \varphi(y-x) d y$. 
Далее, в нашем случае в силу теоремы Фубини и неравенства Гёльдера имеем

$$
\begin{aligned}
d_{0}(f)^{2}=\int_{X^{2}}|f(x)||f(y)||m|(d x, d y) & \leqslant \int_{X^{2}}|f(x)||f(y)| \varphi(x-y) d x d y \\
& \leqslant \int_{X} f(x) J(x) d x \leqslant\|f\|_{r}\|J\|_{r /(r-1)}
\end{aligned}
$$

Остается положить в $(16) r=2 p /(2 p-1)$ и $q=r /(r-1)=2 p$. Предложение доказано.

Рассмотрим, например, так называемое фрактальное броуновское движение с параметром Херста $h>\frac{1}{2}$ (это так называемое регулярное фрактальное броуновское движение), т.е. центрированный гауссовский процесс $\{B(t), t \in[0, T]\}$ с ковариационной функцией

$$
\Phi_{h}(t, s):=\mathbf{E} B(t) B(s)=\frac{1}{2}\left(s^{2 h}+t^{2 h}-|t-s|^{2 h}\right) .
$$

Заметим, что вне диагонали квадрата $[0, T]^{2}$ ковариационная мера $m$ имеет полсжительную плотность $h(2 h-1)|t-s|^{2 h-2}$ относительно меры Лебега. Ее можно вычислить, взяв вторую смешанную производную от $\Phi_{h}(t, s)$ при $t \neq s$.

Кроме того, сужение $m$ на диагональ будет нулевым, так как мера квадрата с ребром $\delta$, «нанизанного» на диагональ, допускает представление

$$
m((t, t+\delta] \times(t, t+\delta])=2 h \delta^{2 h}+O\left(\delta^{2}\right),
$$

т.е. она пропорциональна $\delta^{1+\varepsilon}$ при $2 h>1$ (см. вычисление ковариационой меры диагонали в первом примере п. 3.2). Таким образом, можно считать, что ковариационная мера всюду будет иметь неотрицательную плотность относительно меры Лебега (т.е. по существу это тот же случай, что и в п. 3.1), причем неограниченную в окрестности диагонали:

$$
\phi(t, s)= \begin{cases}h(2 h-1)|t-s|^{2 h-2}, & t \neq s, \\ 0, & t=s .\end{cases}
$$

Легко видеть, что эта плотность интегрируема в любой положительной степени $p$, строго меньшей $1 /(2-2 h)$.

Таким образом, мы получили достаточное условие принадлежности функции $f$ пространству $\left(S, d_{0}\right)$ в виде вложения пространств $L_{r}(X, d t)$ в $\left(S, d_{0}\right)$ для любого $r>1 / h$, поскольку при $p \uparrow p_{0}:=1 /(2-2 h)$ в силу монотонности отношения $2 p /(2 p-1)$ имеем

$$
r=\frac{2 p}{2 p-1} \downarrow \frac{2 p_{0}}{2 p_{0}-1}=\frac{1}{h} .
$$

Отметим, что для данного частного примера несколько более точное вложение $L_{1 / h}(X, d t)$ в $\left(S, d_{0}\right)$ установлено в [9]. Кроме того, ввиду 
очевидной аналогии с примером в п. 3.1 легко выписывается и условие существования кратного стохастического интеграла, построенного по приращениям регулярного фрактального броуновского движения, которое уже приведено в (7), где надо положить

$$
\bar{q}\left(t_{1}, \ldots, t_{2 k}\right)=\sum \prod \phi\left(t_{i}, t_{j}\right)
$$

здесь сумма берется по всевозможным разбиениям множества индексов $\{1, \ldots, 2 k\}$ на пары, а произведение - по всем таким парам в фиксированном разбиении.

\section{СПИСОК ЛИТЕРАТУРЫ}

1. Wiener N. Differential space. - J. Math. Phys., 1923, v. 2, p. 131-179.

2. Колмогоров $A$. H. Кривые в гильбертовском пространстве, инвариантные по отношению к однопараметрической группе движений. - Докл. АН СССР, 1940, т. 26 , № 1 , c. $6-9$.

3. Cramér H. On the theory of stationary random processes. - Ann. Math., 1940, v. 41, p. 215-230.

4. Itô K. Stochastic integral. - Proc. Imp. Acad. Tokyo, 1944, v. 20, p. 519-524.

5. Major P. Multiple Wiener-Itô integrals. - Lecture Notes in Math., 1981, v. 849, p. 1-127.

6. Лоэв М. Теория вероятностей. М.: ИЛ, 1962, 719 c.

7. Cambanis S., Huang S.T. Stochastic and multiple Wiener integrals for Gaussian processes. - Ann. Probab., 1978, v. 6, № 4, p. 585-614.

8. Dasgupta A., Kallianpur G. Multiple fractional integrals. - Probab. Theory Relat. Fields, 1999, v. 115, № 4, p. 505-525.

9. Pipiras V., Taqqu M.S. Integration questions related to fractional Brownian motion. - Probab. Theory Relat. Fields, 2000, v. 118, № 2, p. 251-291.

10. Alòs E., Mazet O., Nualart D. Stochastic calculus with respect to Gaussian processes. - Ann. Probab., 2001, v. 29, № 2, p. 766-801.

11. Гихман И. И., Скороход А.В. Введение в теорию случайных процессов. М.: Наука, 1965,656 с.

12. Колмогоров A.H., Фомин C.B. Элементы теории функций и функционального анализа. М.: Наука, 1981, 543 с.

13. Боровков А. А. Теория вероятностей. М.: Эдиториал УРСС, 1999, 472 с.

14. Биллингсли П. Сходимость вероятностных мер. М.: Наука, 1977, 352 с.

15. Борисов И.С. Об одном критерии марковости гауссовских случайных процессов. - Теория вероятн. и ее примен., 1982 , т. 27 , в. 4, с. 802-805.

16. Белов О.В., Ильин В. П., Никольский С. М. Интегральные представления функций и теоремы вложения. М.: Наука, 1996, 480 c. 